\title{
Long-term Survival With Afatinib in a Patient With Lung Adenocarcinoma Harboring Double Uncommon EGFR L861Q and G719X Mutations
}

\author{
GEN OHARA ${ }^{1}$, SHINICHIRO OKAUCHI ${ }^{1}$, YUIKA SASATANI ${ }^{1}$, TOSHIHIRO SHIOZAWA ${ }^{2}$, \\ HIDEYASU YAMADA ${ }^{3}$, KUNIHIKO MIYAZAKI ${ }^{4}$ and HIROAKI SATOH ${ }^{1}$ \\ ${ }^{1}$ Division of Respiratory Medicine, Mito Medical Center, \\ University of Tsukuba-Mito Kyodo General Hospital, Mito, Japan; \\ ${ }^{2}$ Division of Respiratory Medicine, Faculty of Medicine, University of Tsukuba, Tsukuba, Japan; \\ ${ }^{3}$ Division of Respiratory Medicine, Hitachinaka Medical Center, \\ University of Tsukuba-Hitachinaka General Hospital, Hitachinaka, Japan; \\ ${ }^{4}$ Division of Respiratory Medicine, Ryugasaki Saiseikai General Hospital, Ryugasaki, Japan
}

\begin{abstract}
Background: In the majority of non-small cell lung cancer (NSCLC) patients with uncommon EGFR mutations, first generation epidermal growth factor receptortyrosine kinase inhibitors (EGFR-TKIs) are ineffective. The second-generation TKI, afatinib, is considered effective in patients with uncommon mutations, however, long-term survivors have been rare. Case Report: We report herein a patient with lung adenocarcinoma harboring double uncommon EGFR L861Q and G719X mutations, who is free of disease 32 months after initiation of afatinib therapy. To our best knowledge, this patient has the longest response among other patients with double uncommon mutations. Conclusion: Patients with this type of NSCLC may obtain long-term survival with afatinib.
\end{abstract}

Clinically, non-small cell lung cancer (NSCLC) patients with rare epidermal growth factor receptor $(E G F R)$ mutations are resistant to first-generation EGFR- tyrosine kinase inhibitors (TKIs). The second-generation EGFR-TKI, afatinib, has binding a stronger affinity to the EGFR receptor compared to first-generation TKI (1). Regarding EGFR-TKI therapy,

This article is freely accessible online.

Correspondence to: Hiroaki Satoh, MD, Ph.D., Division of Respiratory Medicine, Mito Medical Center, University of TsukubaMito Kyodo General Hospital, Miya-machi 3-2-7, Mito-city, Ibaraki, 310-0015, Japan. Tel: +81 292312371, e-mail: hirosato@md.tsukuba.ac.jp

Key Words: Long-term survival, afatinib, lung adenocarcinoma, uncommon EGFR mutations. two issues are currently being discussed. One is, in which order to give each TKI when treating cells with sequential TKIs in order to improve overall survival (2). The other is which TKI to select for patients with uncommon EGFR mutations (3). The efficacy of afatinib in NSCLC patients with uncommon EGFR mutations has been confirmed in clinical trials (3). As there are several types of uncommon EGFR mutations, it is necessary to evaluate the effect of afatinib for each mutation type. In order to assess the efficacy of afatinib treatment, information from case reports is important. This is one of the most important methods to evaluate treatment for orphan diseases. In fact, there have been case reports of patients who have responded to afatinib, and who have survived for a long period of time (4-14). We herein describe the case of a patient with lung adenocarcinoma harboring double uncommon EGFR L861Q and G719X mutations, who is free of disease 32 months after initiation of afatinib therapy. To our best knowledge, this patient has the longest response known among patients carrying double uncommon mutations.

\section{Case Report}

A 65-year-old man was referred to our hospital with complaints of right chest pain and general fatigue. He was a 30 pack-year smoker. Chest CT scan revealed a tumor in the left lung with enlargement of bilateral mediastinal lymph nodes (Figure 1). Physical examination was normal except for enlargement of the right cervical lymph nodes. Biopsy specimens from the cervical lymph nodes were obtained, and the patient was diagnosed as having lung adenocarcinoma. Examination of the DNA sequence of the EGFR gene revealed the uncommon EGFR L861Q and G719X 

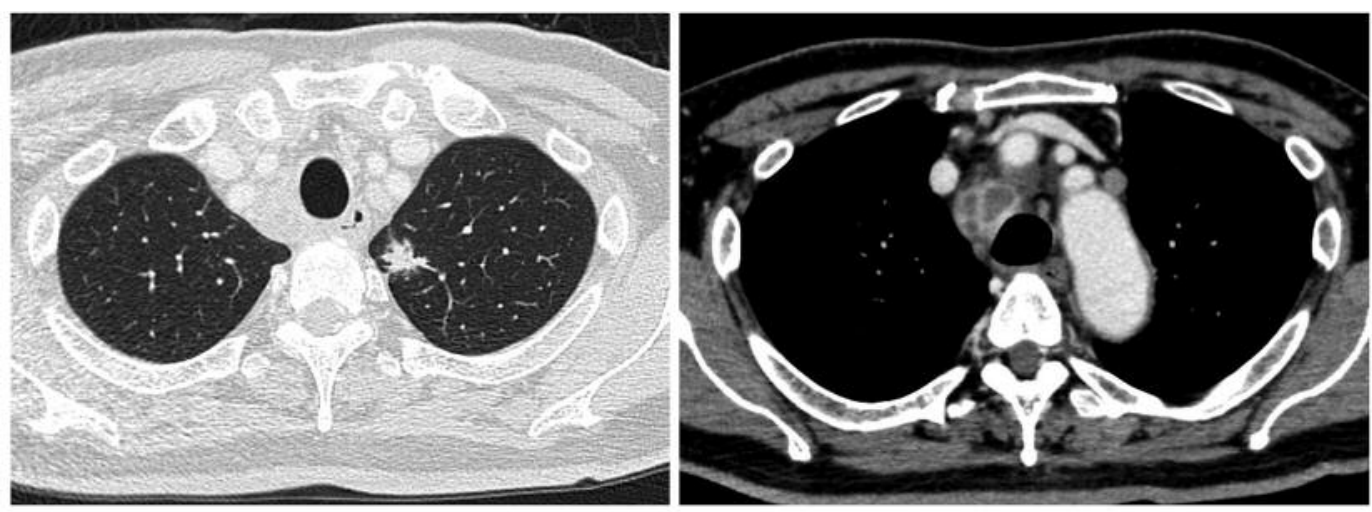

Figure 1. Chest radiograph revealed a tumor $3 \mathrm{~cm}$ in diameter in the left lung with enlargement of bilateral mediastinal lymph nodes.

mutations. Chest and abdominal computed tomography (CT) scan, brain magnetic resonance imaging (MRI), and bone scan showed multiple rib metastases in both sides (Figure 2). Clinical stage was T1cN3M1c (LYM, OSS) stage IVB. The patient received afatinib (orally $80 \mathrm{mg} /$ day). Chest CT scan taken one month after the initiation of afatinib revealed shrinkage of the primary lesion in the left lung and bilateral mediastinal lymph nodes (Figure 3). No severe adverse effects were observed, except for grade II skin toxicity. CT scans performed 30 months after the initiation of therapy revealed no recurrence. The patient is still well 32 months after the initiation of afatinib with no recurrence.

\section{Discussion}

Herein, we report a 32-month responder with lung adenocarcinoma harboring double uncommon EGFR L861Q and G719X mutations, who was treated with afatinib therapy. The clinical activity of afatinib in NSCLC patients with uncommon EGFR mutations has been confirmed in clinical trials (3). This study was a combined post-hoc analysis of three clinical trials, LUX-Lung 2, LUX-Lung 3, and LUX-Lung 6, in patients with advanced NSCLC harboring uncommon EGFR mutations (3). In the analysis, 75 (12\%) of 600 patients, who were treated with afatinib, had uncommon EGFR mutations (3). Median progression-free survival of the three uncommon mutation groups: point mutations or duplications in exons 1821, de novo Thr790Met mutations in exon 20 alone or in combination with other mutations, or exon 20 insertions, was 10.7, 2.9, and 2.7 months, respectively. Median overall survival was $19.4,14.9$, and 9.2 months, respectively (3). It is well known that there are many types of uncommon mutations (314). In addition, the existence of patients with multiple uncommon mutations has been clarified (3-14). Since the number of patients with each uncommon mutation type is small and there are many types of uncommon mutations at the gene level, it is difficult to clarify the PFS and OS of each type of
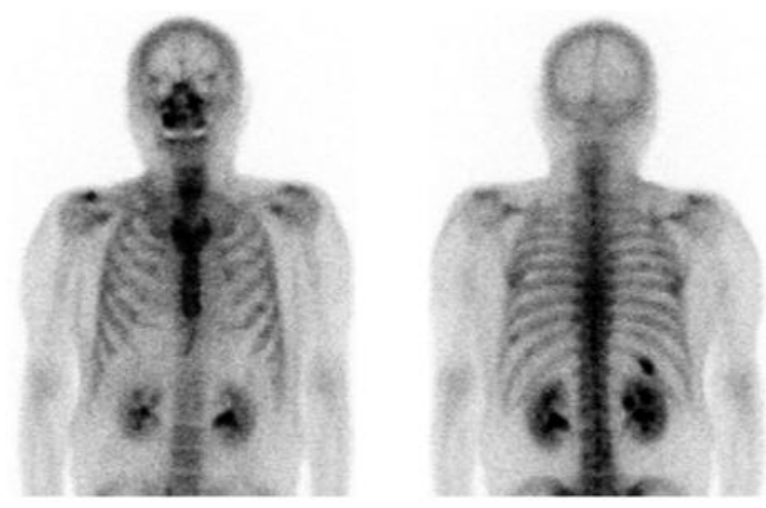

Figure 2. Bone scan revealed multiple rib metastases in both sides.

patients with uncommon mutations. Given these backgrounds, case reports on the treatment of each type of uncommon mutation patient should be important. This is one of the important methods to evaluate treatment for orphan diseases. With regard to afatinib therapy in patients with lung adenocarcinoma harboring uncommon EGFR mutations, there were 11 case reports (4-14). In most case reports, afatinib was given as a first-line therapeutic drug $(4-12,14)$. Most cases had a PFS of less than one year $(5-7,9,10,14)$, but some patients had a PFS of more than one year $(8,11,13)$. An et al. reported a case with a great efficacy of afatinib on a patient with lung adenocarcinoma harboring uncommon EGFR delE709_T710insD mutations. According to the Authors of this study, PFS of the patient was 11 months and the overall survival exceeded 21 months (5). Čoupková and Vyzula reported on a patient with EGFR (in exon 18-T179X) mutation persistent PFS for 19 months (8). Watanabe et al. showed good response without progression for 12 months in a patient with G719X and S768I mutations (12). Chan et al. presented a patient with a PFS of 37 months and of 54 months after a second-line afatinib therapy (11). This patient had EGFR exon 20 insertion mutation 

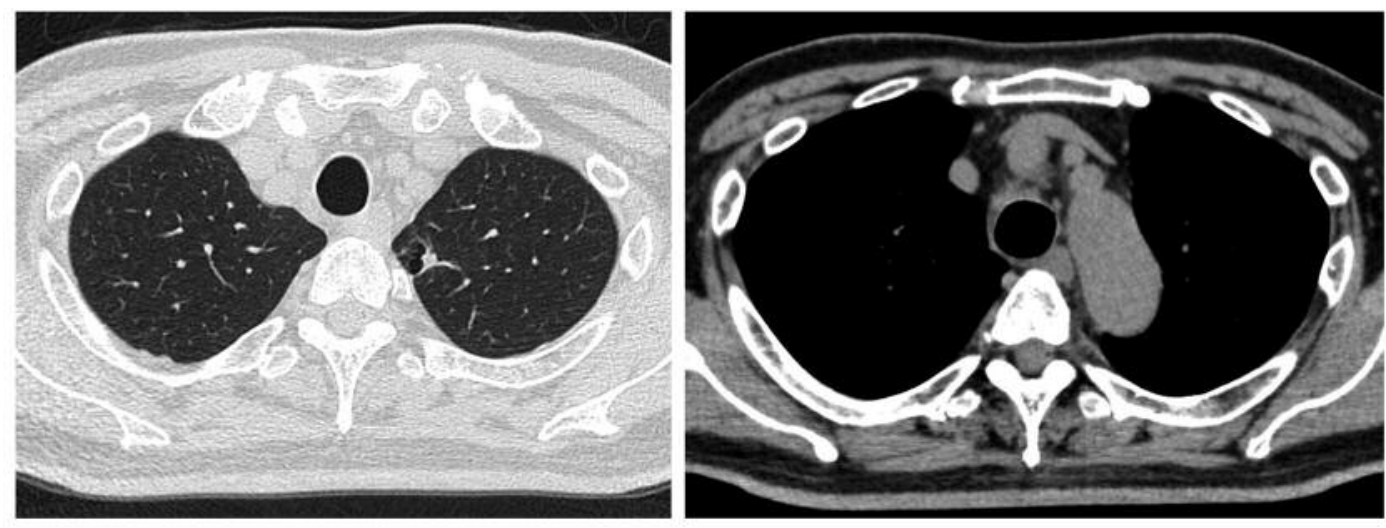

Figure 3. Chest CT scan taken one month after the initiation of afatinib revealed shrinkage of the primary lesion in the left lung and of the bilateral mediastinal lymph nodes.

(11). In these case reports, as described above, EGFR mutation of the patients was heterogeneous (4-14). Among these case reports, interestingly, there was one report of a patient with the same double uncommon EGFR L861Q and G719X mutations as observed in our patient (14). In this report by Kimura et al., the effectiveness of afatinib for this patient with the double uncommon mutation, was confirmed by using a special visual assay (14). Also, this report showed that the PFS of the patient was 'more than 10 months' (14). To our best knowledge, therefore, this is a case report of the longest survival of an afatinib responder with lung adenocarcinoma harboring double uncommon EGFR L861Q and G719X mutations. While there were reports of patients who had a therapeutic effect and those who had a long survival (5-14), there are possibly many patients who had no response and a short survival (15). This is a publication bias. Of course, clinical trials cannot clarify 'the truth' due to the small number of patients. Although the 'evidence level' is low, it is important to carefully examine the information obtained from case reports and to collect and analyze information from case reports. Our results suggest that patients with lung adenocarcinoma harboring double uncommon EGFR L861Q and G719X mutations might have long-term survival, if the suitable treatment is provided.

\section{Ethics Statement}

This study was approved by the institutional Ethics Committee of each Hospital (approval number: NO16-66). Written comprehensive informed consent for obtaining pathological specimens at the time of admission was obtained from the patient.

\section{Conflicts of Interest}

The Authors have no conflicts of interest to declare regarding this study.

\section{Authors' Contributions}

GO and HS designed the study. GO, SO and YS, collected the data. TS, HY, KM and HS analyzed the data and prepared the article. All Authors approved the final version of the article.

\section{References}

1 Solca F, Dahl G, Zoephel A, Bader G, Sanderson M, Klein C, Kraemer O, Himmelsbach F, Haaksma E and Adolf GR: Target binding properties and cellular activity of afatinib (BIBW 2992), an irreversible ErbB family blocker. J Pharmacol Exp Ther 343(2): 342-350, 2012. PMID: 22888144. DOI: 10.1124/jpet. 112.197756

2 Tamiya M, Tamiya A, Suzuki H, Moriizumi K, Nakahama K, Taniguchi Y, Kunimasa K, Kimura M, Inoue T, Kuhara H, Nishino K, Hirashima T, Atagi S, Imamura F and Kumagai T: Which is better EGFR-TKI followed by osimertinib: afatinib or gefitinib/erlotinib? Anticancer Res 39(7): 3923-3929, 2019. PMID: 31262922. DOI: 10.21873/anticanres.13544

3 Yang JC, Sequist LV, Geater SL, Tsai CM, Mok TS, Schuler M, Yamamoto N, Yu CJ, Ou SH, Zhou C, Massey D, Zazulina V and $\mathrm{Wu}$ YL: Clinical activity of afatinib in patients with advanced non-small-cell lung cancer harbouring uncommon EGFR mutations: a combined post-hoc analysis of LUX-Lung 2, LUXLung 3, and LUX-Lung 6. Lancet Oncol 16(7): 830-838, 2015. PMID: 26051236. DOI: 10.1016/S1470-2045(15)00026-1

4 Iida Y, Kumasawa F, Shimizu T, Shintani Y, Takahashi N and Gon Y: Successful treatment of an elderly patient with an uncommon L861Q epidermal growth factor receptor mutation with low-dose afatinib: A case report. Thorac Cancer, 2019. PMID: 31779047. DOI: 10.1111/1759-7714.13269

5 An N, Wang H, Zhu H, Yan W, Jing W, Kong L, Zhang Y and Yu J: Great efficacy of afatinib on a patient with lung adenocarcinoma harboring uncommon EGFR delE709_T710insD mutations: a case report. Onco Targets Ther 12: 7399-7404, 2019. PMID: 31686847. DOI: $10.2147 /$ OTT.S221638

6 Ma C, Huang C, Tang D, Ye X, Li Z, Liu R, Mu N, Li J, Jiang $\mathrm{R}$ and Zhang $\mathrm{J}$ : Afatinib for Advanced non-small cell lung cancer in a case with an uncommon epidermal growth factor 
receptor mutation (G719A) Identified in the cerebrospinal fluid Front Oncol 9: 628, 2019. PMID: 31396478. DOI: 10.3389/ fonc. 2019.00628

7 Cai Y, Wang X, Guo Y, Sun C, Xu Y, Qiu S and Ma K: Successful treatment of a lung adenocarcinoma patient with a novel EGFR exon 20-ins mutation with afatinib: A case report. Medicine (Baltimore) 98(1): e13890, 2019. PMID: 30608413. DOI: 10.1097/MD.0000000000013890

8 Čoupková $\mathrm{H}$ and Vyzula R: Afatinib in the Treatment of advanced non-small cell lung cancer with rare EGFR (in exon 18-T179X) mutation - a case report. Klin Onkol 31(5): 380-383, 2018. PMID: 30541326. DOI: 10.14735/amko2018380

9 Qin BD, Jiao XD, Yuan LY, Liu K, Wang Z, Qin WX and Zang YS: The effectiveness of afatinib and osimertinib in a Chinese patient with advanced lung adenocarcinoma harboring a rare triple EGFR mutation (R670W/H835L/L833V): a case report and literature review. Onco Targets Ther 11: 4739-4745, 2018. PMID: 30127622. DOI: 10.2147/OTT.S167346

10 Duan H, Peng Y, Cui H, Qiu Y, Li Q, Zhang J, Shen W, Sun C and Luo C: Effectiveness of afatinib after ineffectiveness of gefitinib in an advanced lung adenocarcinoma patient with a single EGFR exon 20 S768I mutation: a case report. Onco Targets Ther 11: 2303-2309, 2018. PMID: 29731638. DOI: 10.2147/OTT.S151125

11 Chan RT: Afatinib for an EGFR exon 20 insertion mutation: A case report of progressive stage IV metastatic lung adenocarcinoma with 54 months' survival. Asia Pac J Clin Oncol 14: 7-9, 2018. PMID: 29508940. DOI: 10.1111/ajco.12853
12 Watanabe M, Oizumi S, Kiuchi S, Yamada N, Yokouchi H, Fukumoto $\mathrm{S}$ and Harada M: The effectiveness of afatinib in a patient with advanced lung adenocarcinoma harboring rare G719X and S768I mutations. Intern Med 57(7): 993-996, 2018. PMID: 29225262. DOI: 10.2169/internalmedicine.9565-17

13 Tamiya M, Shiroyama T, Nishihara T, Nishida T, Hayama M, Tanaka A, Morishita N, Suzuki H, Okamoto N and Hirashima T: Afatinib successfully treated leptomeningeal metastasis during erlotinib treatment in a patient with EGFR-mutant (Exon18:G719S) lung adenocarcinoma as a second-line chemotherapy. Asia Pac J Clin Oncol 13(5): e531-e533, 2017. PMID: 28004883. DOI: 10.1111/ajco.12643

14 Kimura S, Tanaka K, Harada T, Liu R, Shibahara D, Kawano Y, Nakanishi Y and Okamoto I: Sensitivity of epidermal growth factor receptor with single or double uncommon mutations to afatinib confirmed by a visual assay. Cancer Sci 109(11): 36573661, 2018. PMID: 30255614. DOI: 10.1111/cas.13787

15 Chen LC, Shih JY, Yu CJ and Yang CY: A rare epidermal growth factor receptor $\mathrm{H} 773 \mathrm{~L} / \mathrm{V} 774 \mathrm{M}$ compound mutation in advanced non-small-cell lung cancer with poor response to epidermal growth factor receptor tyrosine kinase inhibitor. Respirol Case Rep 7(5): e00425, 2019. PMID: 31007929. DOI: 10.1002/ rer 2.425

Received March 9, 2020

Revised March 23, 2020

Accepted March 24, 2020 